\title{
EFFECTS OF PROPHYLACTIC MATERNAL DEXAMETHASONE ADMINISTRATION ON THE NEONATAL RESPIRATORY OUTCOMES AT TERM AFTER ELECTIVE CAESAREAN SECTION: RANDOMIZED CONTROLLED TRIAL
}

\author{
Hasina Sadiq ${ }^{1 \otimes}$, Irum Sohail'
}

\begin{abstract}
OBJECTIVE: To assess the effects of prophylactic antenatal dexamethasone administration in reducing the neonatal respiratory morbidity at term after elective lower segment caesarean section (LSCS).

METHODS: This open label randomized-controlled trial was conducted in Department of Obstetrics and Gynaecology, Kahuta Research Laboratories (KRL) Hospital, Islamabad from September 2017 to February 2018. Patients with singleton pregnancy and non-anomalous fetus between gestational age of $37+{ }^{\circ}$ and $38+^{6}$, planned to be delivered by elective LSCS were randomized through block-randomization method into interventional group $(A)$ : who received dexamethasone and control group (B): who did not receive prophylactic dexamethasone. Main outcomes of the study included Apgarscore at one \& five minutes, admission to neonatal intensive care unit (NICU) due to transient tachypnea of newborn (TTN) and respiratory distress syndrome (RDS). Data was analyzed by SPSS version 23.
\end{abstract}

RESULTS: A total of 304 women were randomized and equally assigned to interventional and control group. In group-A, 2 neonates developed RDS as compared to one neonate in group- $B(p=0.391)$. Mean age of patients in group $A$ and $B$ was $30.48 \pm 4.18$ years and $29.38 \pm 4.57$ years respectively. Fiveminutes Apgar score was $8.91 \pm 0.290$ in group $A \& 8.87 \pm 0.393$ in group $B$ $(p=0.320)$. TTN developed in four neonates in group-A while one neonate in group-B $(p=0.176)$. Eighteen $(11.8 \%)$ cases in group A \& $12(7.9 \%)$ cases in group B were admitted in NICU $(p=0.249)$.

CONCLUSION: Prophylactic dexamethasone administration in term LSCS after 37 weeks of gestational age has no significant effects on reducing the incidence of RDS, TTN and neonatal admission to NICU.

KEY WORDS: Dexamethasone (MeSH); Cesarean Section (MeSH); Respiratory Distress Syndrome (MeSH); Transient Tachypnea of Newborn (MeSH); Randomized Controlled Trial (MeSH); Apgar Score (MeSH); Infant, Newborn (MeSH); Intensive Care Units, Neonatal $(\mathrm{MeSH})$; Prenatal Care (MeSH); Elective Surgical Procedures $(\mathrm{MeSH})$.

THIS ARTICLE MAY BE CITED AS: Sadiq H, Sohail I. Effects of prophylactic maternal dexamethasone administration on the neonatal respiratory outcomes at term after elective caesarean section: randomized controlled trial. Khyber Med Univ J 2019;II(I): 6-I.

\section{INTRODUCTION}

W orldwide, lower segment caesarean section (LSCS) rate is increasing due to multiple reasons including breech presentation, previous caesarean section, placenta previa maternal anxiety, maternal own request and suspected fetal macrosomia. ${ }^{1,2}$ The caesarean section delivery is believed to be associated with maternal and neonatal complications as compared to

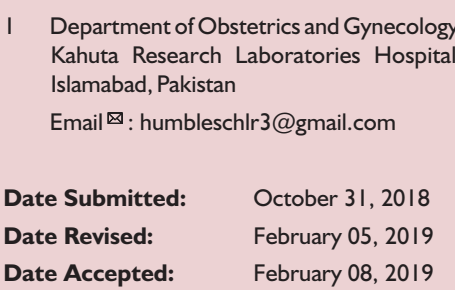

vaginal delivery. ${ }^{3}$ LSCS done before 39 weeks of gestational age is linked with increased respiratory morbidity' requiring admission to neonatal intensive care unit (NICU) and hence increasing cost on health services providers or on the parents. Caesarean is also associated with abnormal immune responses that lead to development of diseases like allergies, asthma, diabetes mellitus type $I$ and celiac disease. ${ }^{4}$

Neonatal respiratory complications like respiratory distress syndrome (RDS) and transient tachypnea of newborn (TTN) are more common in infants delivered via LSCS as compared to those delivered vaginally. Studies have shown that about $50 \%$ of neonates with RDS were due to the iatrogenic early term caesarean section. ${ }^{5,6}$ Apart from mode of delivery, the gestational age at the time of delivery also affects the neonatal respiratory outcomes. ${ }^{5}$ Whereas, the risk is more in neonates born between 34 to 36 weeks of gestational age as compared to those born at term. ${ }^{7}$ The risk of neonatal respiratory complications is approximately 1.7 times when born between $37+{ }^{\circ}$ to $37+{ }^{6}$ weeks as compared to those between $38+0$ to $38+{ }^{6}$ weeks in whom the risk is about 2.4 times relatively more than those born after $39+{ }^{\circ}$ weeks. ${ }^{5}$ Therefore, it is recommended for elective LSCS to be delayed up till 39 weeks of gestational age until seemed indispensable.

In order to reduce the respiratory complications, antenatal prophylactic corticosteroids are given as they accelerate the lung maturation and surfactant production. ${ }^{7}$ Various types of steroids are available such as dexamethasone, betamethasone and hydrocortisone. Dexamethasone which is a long acting steroid, is widely available and cheap. It is given as a single course consisting of 2 doses of $12 \mathrm{mg}$ 


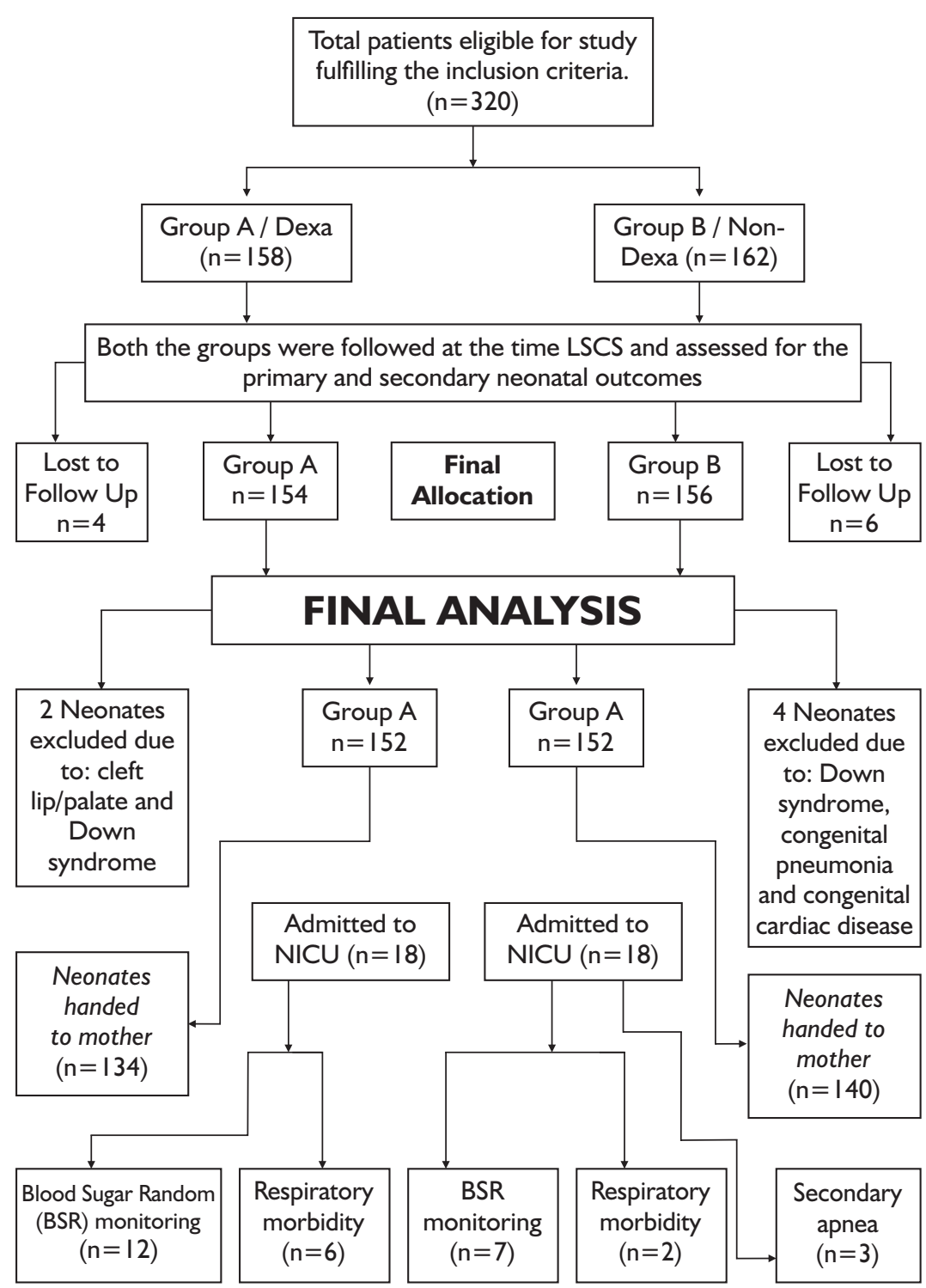

Figure I: Methodology Flow Chart

intramuscularly 12 hours apart. Their maximum effects are observed when delivery done 24 hours after and up to I week from the second dose. ${ }^{8}$ Their benefits are extensively observed in preterm deliveries between 24 to 34 weeks of gestational age. ${ }^{7}$ But their role in late preterm and term fetuses is still questionable. They are associated with immediate side effects like neonatal hypoglycemia and long term adverse effects on metabolic, neurological and cognitive functions. ' Steroids exposure at this stage affects the neuronal growth and development in the fetal brain, consequently varying their migration, synaptic transmission and interferes with neurotransmitter actions. The mechanism proposed in animal studies is possibly by the apoptosis and cell death therefore limiting the neuronal growth and function. ${ }^{10}$ Due to this, the gestational age until which the steroids should be given is never agreed upon and their use beyond 34 weeks is still controversial. ${ }^{7}$ The Royal College of Obstetricians and Gynaecologists (RCOG) recommends the antenatal corticosteroids for all women undergoing planned elective caesarean before $38+{ }^{6}$ weeks of gestational age. ${ }^{10}$ However, American College of Obstetricians and Gynecologists does not recommend antenatal corticosteroids for planned term LSCS," as the committee opinion strongly recommend the single course for imminent preterm deliveries. But due to lack of sufficient evidence regarding long term outcomes on neurodevelopment of neonate, steroids for term deliveries will depend upon the condition and is not contraindicated. We carried out this study to see the respiratory effects of prophylactic dexamethasone before early term elective LSCS as compared to usual management without steroids in our population.

\section{METHODS}

This was a prospective open label, randomized controlled interventional study. Ethical approval was obtained from hospital research evaluation committee. The sample size calculated on the basis of World Health Organization (WHO) sample size criteria was 89 patients in each group for statistically significant results. But for better results we have included more patients in both groups. The study was conducted over a period of six months spanning from Sep, 2017 to Feb, 2018 at Kahuta Research Laboratories (KRL) Hospital, Islamabad. Structured questionnaire was designed for data collection including information regarding the maternal data, use of dexamethasone, gestational age at the time of LSCS, neonatal data, weight, gender and Apgar score.

The patients were enrolled from the Obstetrics Ward and it included pregnant women with singleton alive fetus between gestational age of $37+^{\circ}$ to $38+{ }^{6}$ weeks, admitted for elective LSCS under spinal anesthesia. gestational age of these patients was confirmed by the date of last menstrual period and in cases of unsure of dates, by the first trimester dating scan. Patients with uncomplicated gestational diabetes mellitus, pregnancy induced hypertension, anemia and asthma were included in both groups. Women with type I or 2 diabetes mellitus, chronic hypertension, preeclampsia, pre-labour rupture of membranes, multiple pregnancies, infections including tuberculosis, congenital anomalous fetus, intrauterine growth restriction 
(IUGR) fetuses and emergency LSCS or operated under general anesthesia were excluded.

After scrutinizing these patients, they were randomly distributed in groups $\mathrm{A}$ and $B$ and informed consent was taken. The randomization was done on the basis of block randomization method. Group A was interventional or Dexa group and received one course of dexamethasone within 7 days of LSCS. One course of dexamethasone contained two doses of $12 \mathrm{mg}$, each given intramuscularly to the pregnant woman, 12 hours apart. Group B was a non-interventional or control group that did not receive dexamethasone injection before LSCS.

For patients with gestational diabetes (GDM), blood sugar - random (BSR) was monitored along with the dose of dexamethasone and regular insulin was given according to sliding scale. All those pregnant patients who had received dexamethasone before 7 days at any time during pregnancy were also excluded from the study. The baseline characteristics of patients like age, parity, history of miscarriage were also noted.

All the patients were pursued in operation theatre, where elective LSCS was performed by the consultant gynecologist or senior resident and the delivered baby was received by the neonatologist. Primary outcomes included assessment of baby by Apgar scores at one and five minutes, the needs for neonatal resuscitation, and admission of baby to NICU. The secondary outcomes included development of RDS or TTN, neonatal hypoglycemia, supplemental oxygen given, use of surfactant, ventilatory support and length of hospital stay. All the variables were noted on the structured performa.

The statistical analysis was performed with SPSS 23.0. Frequency and percentage was computed for qualitative variables and chi-square test was applied for analysis. Mean \pm standard deviation was calculated for quantitative variables and then analyzed by Independent Samples $t$ Test, keeping the level of significance “p" value below 0.05 .

\section{RESULTS}

A total of 320 patients were studied, and divided into groups "A" and "B". Group A consisting of 158 patients while group $B$ of 162 patients. Birth weight of infants was $2.90 \pm 0.410 \mathrm{~kg}$ in group A \& $2.95 \pm 0.332 \mathrm{~kg}$ in group $B$.

The baseline characteristics of both groups were comparable with each other and included age, gestational age at the time of delivery, parity, previous miscarriages, medical comorbids consisting of gestational hypertension, GDM, anemia and asthma. Mean age of patients in group $A$ and $B$ was $30.48 \pm 4.18$ years and $29.38 \pm 4.57$ years respectively. The gestational age at the time of delivery was $37.9 \pm 0.5$ I weeks for group $A$ and for group $B$ was $37.5 \pm 0.48$ weeks. Majority of the patients were para I and with no history of miscarriages in both groups.

TABLE I: PRIMARY NEONATAL OUTCOMES

\begin{tabular}{|l|l|c|c|c|}
\hline \multicolumn{2}{|c|}{ Variables } & $\begin{array}{c}\text { Group A } \\
\text { Dexa Group } \\
(\mathbf{n}=152)\end{array}$ & $\begin{array}{c}\text { Group } \\
\text { Control Group } \\
(\mathbf{n}=152)\end{array}$ & p-value \\
\hline \multirow{2}{*}{ Apgar Score** } & $\mathrm{I}$ minute & $7.48 \pm 0.559$ & $7.32 \pm 0.592$ & $0.09 \mid$ \\
\cline { 2 - 5 } & 5 minutes & $8.91 \pm 0.290$ & $8.87 \pm 0.393$ & 0.320 \\
\hline Neonatal Resuscitation * & $3(2 \%)$ & $3(2 \%)$ & I \\
\hline Admission to NICU* & $18(1 \mathrm{I} .8 \%)$ & $12(7.9 \%)$ & 0.249 \\
\hline
\end{tabular}

* $\mathrm{n}(\%)$; ** mean $\pm \mathrm{SD}$

TABLE II: SECONDARY NEONATAL OUTCOMES

\begin{tabular}{|c|c|c|c|c|}
\hline \multicolumn{2}{|c|}{ Variables } & $\begin{array}{c}\text { Group A } \\
\text { Dexa Group } \\
(n=152)\end{array}$ & $\begin{array}{c}\text { Group B } \\
\text { Control Group } \\
(n=152)\end{array}$ & p-value \\
\hline \multicolumn{2}{|l|}{ Admission to NICU* } & $18(11.8 \%)$ & $12(7.9 \%)$ & 0.249 \\
\hline \multicolumn{2}{|c|}{ Transient Tachypnea of Newborn } & $4(2.6 \%)$ & $\mathrm{I}(0.7 \%)$ & 0.176 \\
\hline \multirow{3}{*}{$\begin{array}{l}\text { Respiratory } \\
\text { Distress Syndrome }\end{array}$} & GRADE I & 0 & $\mathrm{I}(0.7 \%)$ & 0.391 \\
\hline & GRADE 2 & $\mathrm{I}(0.7 \%)$ & 0 & \\
\hline & GRADE 3 & $\mathrm{I}(0.7 \%)$ & 0 & \\
\hline \multicolumn{2}{|c|}{ Supplementary Oxygen } & $4(2.6 \%)$ & $2(1.3 \%)$ & 0.410 \\
\hline \multicolumn{2}{|c|}{ Mechanical Ventilation } & $2(1.3 \%)$ & 0 & 0.156 \\
\hline \multicolumn{2}{|l|}{ Surfactant Given } & $2(1.3 \%)$ & 0 & 0.156 \\
\hline \multirow{2}{*}{$\begin{array}{l}\text { Blood Sugar } \\
\text { Monitoring }\end{array}$} & GDM*** & II (7.2\%) & $7(4.6 \%)$ & 0.236 \\
\hline & Non-GDM & $\mathrm{I}(0.7 \%)$ & 0 & \\
\hline \multirow{4}{*}{$\begin{array}{l}\text { Length of Stay } \\
\text { in NICU }\end{array}$} & Less than 24 hours & $16(10.5 \%)$ & $\mathrm{II}(7.2 \%)$ & 0.546 \\
\hline & 24 to 48 hours & 0 & 0 & \\
\hline & 3 to 7 days & $\mathrm{I}(0.7 \%)$ & $\mathrm{I}(0.7 \%)$ & \\
\hline & $>7$ days & $\mathrm{I}(0.7 \%)$ & 0 & \\
\hline
\end{tabular}

*Neonatal intensive care unit; **Gestational Diabetes Mellitus
Pregnancy induced hypertension $(\mathrm{PIH})$ in group $A$ and $9(5.9 \%)$ in group $B$. GDM was present in about II (7.2\%) and $9(5.9 \%)$ patients in group $A$ and $B$ respectively. One $(0.7 \%)$ patient with severe anemia was present in group A. There was $5(3.3 \%)$ patients of asthma in group $A$ and $I(0.7 \%)$ in group $B$. The patients were followed in the operation theater until the delivery of baby to assess the primary and secondary outcomes. However, 10 patients had incomplete data, 4 from the group $A$ and 6 from group $B$ and were therefore excluded. Six more patients were also excluded as their neonates were diagnosed after birth with congenital conditions. Two neonates in group A were excluded with cleft lip/palate and Down syndrome. While in group $B$, four neonates were excluded (one each with congenital pneumonia, Down was present in about I5(9.9\%) patients 
syndrome with cardiac disease, \& two with cleft lip and palate). So, 304 patients were finally analyzed with equal number of I 52 patients in each group, as shown in study methodology in Figure I.

There were no significant differences in the primary outcomes like neonatal weight at birth, Apgar score at I and 5 minutes, neonatal resuscitation and NICU admission in both groups, as shown in Table I. In group A, two babies developed RDS grade 2 and 3 respectively. Both admitted to NICU for 7 days and received supplementary oxygen, mechanical ventilatory support and surfactant. Both of them were alive at the time of discharge. Four babies developed TTN, all were observed in NICU and handed over within few hours. While in group $B$, only one baby developed TTN, was admitted to NICU and kept under observation, then handed over within few hours. One baby developed RDS grade I, admitted to NICU. Baby received supplementary oxygen and length of NICU stay was 48 hours. The respiratory morbidity was not statistically significant between both groups and not occurred in GDM patients.

As per hospital protocol all neonates born to GDM mothers were admitted in NICU for observation and none of them developed hypoglycemia. All were observed and then handed over. But only one neonate born to mother in Dexa group with no known medical comorbid developed neonatal hypoglycemia. No significant difference found in other parameters like supplementary oxygen, surfactant use and mechanical ventilation between both groups as shown in Table II.

Total neonates admitted to NICU in both groups were not statistically significant. In group A, 18 neonates admitted in NICU, 4 with TTN and 2 with RDS, I I babies with GDM mothers and one baby with non-GDM mother had blood sugar monitoring.In group $B$, twelve neonates admitted, two with respiratory morbidity, seven babies with GDM mothers for blood sugar monitoring and three with secondary apnea revived after neonatal resuscitation for observation.

\section{DISCUSSION}

Steroids are believed to reduce the occurrence of RDS in neonates born before 34 weeks of gestational age but in late preterm and term fetuses the incidence of RDS is less approximately up to $1 \% .{ }^{2}$ The main problem in these neonates is TTN that occurs because of delayed absorption of fluid from the pulmonary alveoli and the steroids may promote the expression of sodium epithelial channels leading to clearance of fluid, but this proposed mechanism has not been accepted universally.12,13 The maximum benefit is achieved when antenatal steroids are given 24 hours to 7 days before the delivery of fetus and this is obtained only in 12 to $25 \%$ of mothers. ${ }^{10}$ Caesarean section has been considered itself to be associated with neonatal respiratory morbidity; TTN more predominantly than RDS in term LSCS. $^{2,6}$

In our study prophylactic dexamethasone administration before early term elective LSCS had no significant effects in reducing the incidence of RDS \& TTN. Cochrane Systemic Review ${ }^{14}$ published in 2009 about prophylactic betamethasone administration 48 hours before caesarean delivery at or after 37 weeks of gestational age showed that there was no statistically significant difference in development of RDS (RR 0.32; 95\% $\mathrm{Cl} 0.07$ to I.58), TTN (RR 0.52; $95 \% \mathrm{Cl}$ 0.25 to I.I I) and need for mechanical ventilation (RR 4.07; $95 \% \mathrm{Cl} 0.46$ to 36.27).

Our study showed no significant effects in reducing the incidence of neonatal admission to NICU in prophylactic dexamethasone administration before early term elective LSCS. These findings are in conflict with results of Cochrane Review showing a statistically significant reduction in NICU admissions for prophylactic betamethasone (RR 0.15; $95 \% \mathrm{Cl} 0.03$ to 0.64 ). Use of different preparation of steroids in our study may be a factor leading to dissimilar results.

A study conducted by Nabhan $A$, et al. ${ }^{15}$ at Egypt about antenatal dexamethasone administration in caesarean deliveries between 34 to 37 weeks of gestational age showed no significant reduction in the occurrence of RDS $(p=0.699)$, TTN $(p=0.612)$, NICU admission $(p=0.535)$ and need for mechanical ventilation $(p=0.509)$. The results are similar to that of our study, as we also didn't find significant differences in any of these neonatal parameters. Similarly, another randomized controlled trial conducted by Porto A, et al. ${ }^{12}$ at Brazil showed no reduction in the incidence of RDS $(p=0.54)$ and TTN $(p=0.77)$ in both the steroid and control groups. There was no significant difference amongst the two groups in need for ventilatory support, exogenous surfactant and type of ventilatory support. Rates of neonatal hypoglycemia was similar in both groups $(p=0.3 \mathrm{I})$. These results are similar to our study but differ in inclusion criteria that it included all deliveries both caesarean and vaginal, between 34 to 36 weeks of gestational age and betamethasone was given prophylactically. There was significant reduction in need of phototherapy in neonatal jaundice in steroid group $(p=0.0 I)$. However, that neonatal parameter was not included in our study.

In contrast to the current study, results of research conducted by Ismail KS, et al. $^{5}$ and Salem MN, et al. ${ }^{16}$ illustrated significant reduction in incidence of neonatal respiratory morbidity and NICU admission in patients given antenatal dexamethasone. Furthermore, Salem MN, et al. ${ }^{16}$ also concluded significant difference in the Apgar score at $I$ and 5 minutes and mechanical ventilation among both groups in comparison to our results. Whereas, results of neonatal gender $(p=0.19)$ and birth weight $(p=0.832)$ were found similar in both studies. Moreover, Ismail KS, et al. ${ }^{5}$ observed that the length of neonatal stay for more than 24 hours was significantly higher in non steroid group, contrary to our results.

Antenatal Steroids for Term Elective Caesarean Trial (ASTEC Trial) ${ }^{17}$ conducted to study the effects of betamethasone after 37 weeks of gestational age showed reduction in incidence of TTN $(R . R=0.54)$ and RDS $(R . R=0.2 I)$ and $50 \%$ reduction in NICU admissions due to respiratory cause. ASTECS trial also included about 10 patients with diabetes mellitus and 
GDM, 4 in steroid group. However, none of their neonate developed respiratory distress that was similar to our results.

There is only one study conducted in Pakistan that is retrospective with a small sample size. Their results are in contrast to our study and showed significant reduction in development of $\operatorname{TTN}(\mathrm{p}=0.005)$ and neonatal admission to NICU $(p=0.044)$. However, length of hospital stay was insignificant $(p=0.18)^{2}$ that was similar to our study $(p=0.546)$.

The TTN is a self-limiting condition, it usually resolves within 72 hours and few babies require intensive level treatment with supplemental oxygen. There is a difference between transitional delay and TTN. Transitional delay is the shorter periods of tachypnea in babies after birth and is self-resolving within a few hours, length varying between 2 to 6 hours. $^{13}$ TTN may be the severe expression of the disease. Therefore, further research should be carried out to investigate the above conditions.

\section{STRENGTH AND LIMITATIONS}

The main strength of this research is that it is a prospective randomized control trial with a reasonable sample size. In the previous studies, medical disorders were excluded, however, in this study, chronic medical diseases were excluded but equal number of patients with gestational hypertension and GDM were included in both groups.

The main limitation of this study is that the stratification of gestational ages was not done and neonatal outcomes were not compared at different gestational age after 37 weeks. Blinding of participants and professionals was not done.

\section{CONCLUSION}

Dexamethasone administration in term LSCS after 37 weeks of gestational age has no significant effects on reducing the incidence of RDS, TTN and neonatal admission. GDM alone is not a risk factor for RDS development. Due to long-term adverse effects of steroids in neonates, they should be judiciously given to pregnant women at term and should not be included in hospital protocols for all term caesarean deliveries

\section{REFERENCES}

I. Mylonas I, Friese K. Indications for and risks of elective cesarean section. Deutsches Ärzteblatt International 2015 Jul; II2(2930):489. DOI: 10.3238/arztebl. 2015.0489

2. Dileep A, Khan NB, Sheikh SS. Comparing neonatal respiratory morbidity in neonates delivered at term by elective Caesarean section with and without dexamethasone: retrospective cohort study. J Pak Med Assoc 20 I 5 Jun;65(6):607- I I.

3. Souza JP, Gülmezoglu AM, Lumbiganon $P$, Laopaiboon $M$, Carroli G, Fawole B, et al. Caesarean section without medical indications is associated with an increased risk of adverse shortterm maternal outcomes: the 20042008 WHO Global Survey on Maternal and Perinatal Health. BMC Med 2010 Nov 10;8:7I. DOI: I0.| I86/I74I-70|5-8-7I.

4. Beogo I, Rojas BM, Gagnon MP. Determinants and materno-fetal outcomes related to cesarean section delivery in private and public hospitals in low-and middle-income countries: a systematic review and meta-analysis protocol. Syst Rev 2017 Jan I4;6(I):5. DOI: I0.I I86/s | 3643-0| 6-0402-6.

5. Ismail KS, Mahgoub AA, Abdulilah K, Elkheir HA, Kholoud AM. Effect of Prophylactic Corticosteroid Therapy on Respiratory Morbidity in Infants Born at Term by Elective Cesarean Section at Omdurman Maternity Hospital. Fam Med Med Sci Res 2017;6(2I5):2. DOI: I0.4172/2327-4972. 1000215 .

6. Hermansen CL, Lorah $\mathrm{KN}$. Respiratory distress in the newborn. Am Fam Physician 2007 Oct;76(7):987-4.

7. Saccone G, Berghella V. Antenatal corticosteroids for maturity of term or near term fetuses: systematic review and meta-analysis of randomized controlled trials. BMJ 2016 Oct;355:i5044. DOI: I0. I I36/ bmj.i5044.

8. Steer PJ, Modi N. Elective caesarean sections-risks to the infant. The Lancet 2009 Aug;374(969I):675-6. DOI: $10.1016 / 50140-6736(09)$ 61544-0

9. Verma RP. Expanded Use of Antenatal Steroid and the Neonate in NICU. J Pediatr Neonatal Care 2016;4(6):00I59. DOI: 10.15406/ jpnc.2016.04.00159.

10. Royal College of Obstetricians and Gynaecologists (RCOG). Antenatal corticosteroids to prevent respiratory distress syndrome. Clinical Green Top Guidelines. Royal College of Obstetricians and Gynaecologists, 2004. [Cited on: October 26, 2018]. Available from URL: https://www.glowm.com/ pdf/Antenatal\%20Corticosteroids $\% 20$ to\%20Reduce\%20Neonatal \%20Morbidity.pdf

II. American College of Obstetricians and Gynecologists' Committee on Obstetric Practice, Society for Maternal - Fetal Medicine. Committee Opinion No.677: antenatal corticosteroids therapy for fetal maturation. Obstet Gynecol 2016; 128(4):el87-94. DOI: 10.1097/AOG.000000000 0001715

12. Porto AM, Coutinho IC, Correia JB, Amorim MM. Effectiveness of antenatal corticosteroids in reducing respiratory disorders in late preterm infants: randomised clinical trial. BMJ $2011 \mathrm{Apr}$ 12;342:dI696. DOI: 10.1136/bmj. dl696.

13. Guglani L, Lakshminrusimha S, Ryan RM. Transient tachypnea of the newborn. Pediatr Rev 2008;29:e5965. DOI: 10.1542/pir.29-I I-e59.

14. Sotiriadis A, Makrydimas G, Papatheodorou S, loannidis J. Corticosteroids for preventing neonatal respiratory morbidity after elective caesarean section at term. Cochrane Database Syst Rev 2009 Oct 7;(4):CD0066I4 DOI: I0.1002/|465 I858.CD0066|4.pub 2. 
EFFECTS OF PROPHYLACTIC MATERNAL DEXAMETHASONE ADMINISTRATION ON THE NEONATAL RESPIRATORY OUTCOMES AT TERM AFTER ELECTIVE CAESAREAN SECTION: RANDOMIZED CONTROLLED TRIAL

15. Nabhan A, Al-Helaly A, Ramadan A. Prophylactic antenatal corticosteroid before elective cesarean delivery at or near term to improve perinatal outcome. Med J Cairo Univ 20 I 4 Mar;82(I):25-8.

16. Salem MN, Abbas AM, Ashry M. Dexamethasone for the prevention of neonatal respiratory morbidity before elective cesarean section at term. Proc Obstet Gynecol 2016; 6(3): I-0.

17. Stutchfield P, Whitaker R, Russell I, Antenatal Steroids for Term Elective Caesarean Section (ASTECS) Research Team. Antenatal betamethasone and incidence of neonatal respiratory distress after elective caesarean section: pragmatic randomized trial. BMJ 2005;331:662. DOI: |0.1|36/ bmj.38547.4I6493.06

\section{AUTHORS' CONTRIBUTIONS}

Following authors have made substantial contributions to the manuscript as under:

HS: Acquisition, analysis \& interpretation of data, drafting the manuscript, final approval of the version to be published.

IS: Concept \& study design, acquisition of data, drafting the manuscript, critical review, final approval of the version to be published.

Authors agree to be accountable for all aspects of the work in ensuring that questions related to the accuracy or integrity of any part of the work are appropriately investigated and resolved.

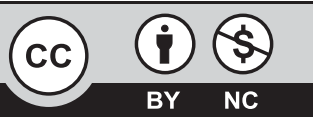

This is an Open Access article distributed under the terms of the Creative Commons Attribution-Non Commercial 2.0 Generic License.

KMUJ web address: www.kmuj.kmu.edu.pk Email address: kmuj@kmu.edu.pk 